\title{
Modeling the Coordinated Movements of the Head and Hand Using Differential Inverse Kinematics
}

\author{
K. Han Kim¹, R. Brent Gillespie ${ }^{2}$ and Bernard J. Martin ${ }^{3}$ \\ ${ }^{1,3}$ HUMOSIM Laboratory, The University of Michigan \\ ${ }^{2}$ Department of Mechanical Engineering, The University of Michigan
}

Copyright @ 2004 SAE International

\begin{abstract}
Hand reach movements for manual work, vehicle operation, and manipulation of controls are planned and guided by visual images actively captured through eye and head movements. It is hypothesized that reach movements are based on the coordination of multiple subsystems that pursue the individual goals of visual gaze and manual reach. In the present study, shared control coordination was simulated in reach movements modeled using differential inverse kinematics. An 8-DOF model represented the torso-neck-head link (visual subsystem), and a 9-DOF model represented the torsoupper limb link (manual subsystem), respectively. Joint angles were predicted in the velocity domain via a pseudo-inverse Jacobian that weighted each link for its contribution to the movement. A secondary objective function was introduced to enable both subsystems to achieve the corresponding movement goals in a coordinated manner by manipulating redundant degrees of freedom. Simulated motions were compared to motion recordings from ten subjects performing right-hand reaches in a seated posture. Joint angles were predicted with and without the contribution of the coordination function, and model accuracy was determined using the RMS error and differences in end posture angles. The results indicated that prediction accuracy was generally better when the coordination function was included. This improvement was more pronounced for low and eccentric targets, as they required greater contribution of the joints shared by both visual and manual subsystems.
\end{abstract}

\section{INTRODUCTION}

Motion prediction models of lifting, reaching, or pointing tasks have been commonly based on a multiple body link system with a single end-effector [1, 2, 3, 4]. However, even simple reaching movements may include multiple task components, other than moving the hand toward the goal target. For example, reaching involves the movement of the head and the eyes to capture images of the environment and build an internal representation of the space in which hand movements are planed and guided. It has been shown that head and/or eye movements are modulated by the movement of the whole body and the hand [5, 6, 7]. In addition, whole body and/or hand movements are also adjusted as a function of visual perception of the environment [8, $9,10]$. Hence it may be suggested that the central nervous system (CNS), while planning and executing a movement, simultaneously coordinates multiple subsystems that pursue individual goals (guiding the hand, displacing the gaze, etc) in order to achieve the general aim of the task (reaching for the target).

Seated reach movements include the movements of the visual sub-system (eye - head - neck - torso), and the manual subsystem (finger - hand - forearm - upper arm - clavicle - torso). Depending on target location and initial posture, both systems may move synergistically (in a same direction) or antagonistically (in different directions) with respect to each other. Since the visual and manual subsystems share a common link (torso), it is hypothesized that the two subsystems negotiate the control of this common link involved in the motion of their respective end-effectors. The CNS should plan and coordinate the movement of each subsystem in order to allocate the use of the common link in such a manner that both subsystems achieve their individual goals. The coordination of multiple subsystems is made possible by manipulating the redundant degrees of freedom of each subsystem.

The aims of the present study are to 1) construct a multibody link system representing the visual and manual subsystems; 2) develop optimization-based inverse kinematics models to simulate the movements of the subsystems separately and integratively; and 3) quantify the benefit of the integration of multiple subsystems by comparing simulated and actual movements. 


\section{METHODS}

\section{DIFFERENTIAL INVERSE KINEMATICS}

The manual subsystem (finger-hand-forearm-upper armclavicle-torso, Figure $1 \mathrm{~A}$ ) and the visual sub-system (eye-head-neck-torso links, Figure 1B) were modeled to represent a human subject performing a seated reach task. The manual and visual subsystems are composed of nine and eight revolute joints, respectively (Table 1).

Table 1. Joint composition of manual and visual subsystem

\begin{tabular}{ll}
\hline \multicolumn{1}{c}{ Manual subsystem joints } & \multicolumn{1}{c}{ Visual subsystem joints } \\
\hline$q_{m 1}$ Torso extension (+) & $q_{v 1}$ Torso extension $(+)$ \\
$q_{m 2}$ Torso lateral bending (+ left) & $q_{v 2}$ Torso lateral bending (+ left) \\
$q_{m 3}$ Torso axial rotation (+ccw) & $q_{v 3}$ Torso axial rotation (+ccw) \\
$q_{m 4}$ Clavicle horizontal (+ forward) & $q_{v 4}$ Neck vertical (+ up) \\
$q_{m 5}$ Clavicle vertical (+ up) & $q_{v 5}$ Neck horizontal (+ left) \\
$q_{m 6}$ Shoulder flexion (+) & $q_{v 6}$ Head extension $(+)$ \\
$q_{m 7}$ Shoulder abduction (+) & $q_{v 7}$ Head tilt (+ left) \\
$q_{m 8}$ Upper arm axial rotation (+ccw) & $q_{v 8}$ Head axial rotation (+ccw) \\
$q_{m 9}$ Elbow flexion $(+)$ & \\
\hline
\end{tabular}

The joint angles of each subsystem are combined and represented by a vector as follows:

$$
\mathbf{q}=\left[\begin{array}{c}
\mathbf{q}_{c} \\
\mathbf{q}_{m} \\
\mathbf{q}_{v}
\end{array}\right]
$$

where

$\mathbf{q}_{c}=\left[q_{1}, q_{2}, q_{3}\right]^{\mathrm{T}} \quad$ (torso joint angles- common in both manual and visual subsystems)

$\mathbf{q}_{m}=\left[q_{m 4}, q_{m 5}, q_{m 6}, q_{m 7}, q_{m 8}, q_{m 9}\right]^{\mathrm{T}} \quad$ (clavicle-shoulderelbow angles: manual subsystem)

$\mathbf{q}_{v}=\left[q_{v 4}, q_{v 5}, q_{v 6}, q_{v 7}, q_{v 8}\right]^{\mathrm{T}}$ (neck-head angles: visual subsystem)

A 9-dof manual subsystem

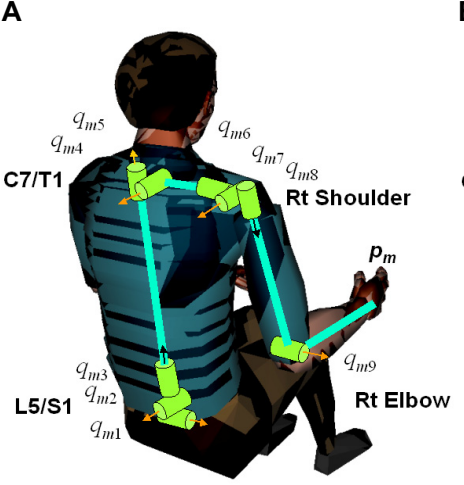

B

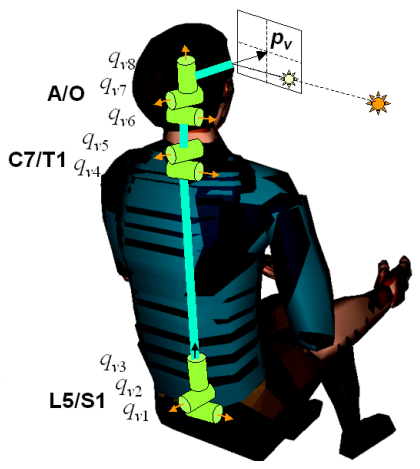

8-dof visual subsystem Figure 1. Multi-link composition of 9-dof manual subsystem (A) and 8dof visual subsystem (B). The arrow extending from each joint indicates the positive direction of joint rotation in a right-hand sense.
The position of the manual subsystem end-effector is represented by Cartesian coordinates as follows:

$$
\mathbf{p}_{m}=\left[f_{1}(\mathbf{q}), f_{2}(\mathbf{q}), f_{3}(\mathbf{q})\right]^{T}
$$

where $f$ denotes a function of direct kinematics of the movement. In contrast, the end-effector of the visual subsystem is defined using a two-dimensional image coordinate system [11].

$$
\mathbf{p}_{v}=\left[\begin{array}{l}
f_{4}(\mathbf{q}) \\
f_{5}(\mathbf{q})
\end{array}\right]=\frac{k}{p_{z}}\left[\begin{array}{l}
p_{x} \\
p_{y}
\end{array}\right]
$$

where the $k$ a spatial scaling factor, and $p_{x}, p_{y}$, and $p_{z}$ represent $x-, y$-, and $z$-coordinates of the target in a head-centered reference frame.

In general, the velocity of the end-effector $\mathbf{p}$ can be obtained by:

$$
\dot{\mathbf{p}}=\frac{\partial f_{i}(\mathbf{q})}{\partial \mathbf{q}} \dot{\mathbf{q}}=\mathbf{J}(\mathbf{q}) \dot{\mathbf{q}}
$$

where $\mathbf{J}$ is a Jacobian matrix. We can use Eq. 3 for both the manual and visual subsystem separately:

$$
\begin{aligned}
& \dot{\mathbf{p}}_{m}=\mathbf{J}_{m} \dot{\mathbf{q}} \\
& \dot{\mathbf{p}}_{v}=\mathbf{J}_{v} \dot{\mathbf{q}}
\end{aligned}
$$

where $\mathbf{J}_{m}$ and $\mathbf{J}_{v}$ represent the Jacobian matrices for the manual and visual subsystem, respectively.

Generally, we wish to obtain $\dot{\mathbf{q}}$ as a function of $\dot{\mathbf{p}}$, but due to the redundant degrees of freedom of the multilink systems, the ordinary inverse of $\mathbf{J}$ cannot be obtained. Alternatively, a weighted pseudo-inverse of $\mathbf{J}$ (denoted as $\mathbf{J}^{\dagger}$ ) may be used.

$$
\dot{\mathbf{q}}=\mathbf{W}^{-1} \mathbf{J}^{T}\left(\mathbf{J W}^{-1} \mathbf{J}^{T}\right)^{-1} \dot{\mathbf{p}}=\mathbf{J}^{\dagger} \dot{\mathbf{p}}
$$

where $\mathbf{W}$ is a weighting matrix that characterizes the instantaneous contribution of each joint. The weighting matrices for the manual subsystem were adapted from an earlier study [2]. For the visual subsystem, regression models of peak velocities of the joint angles, whose measurements are described below, were used to estimate the weighting matrices. Hence, Eq. 5 attempts to satisfy the primary objectives of 1) obtaining joint angles that place the end-effector at the desired positions at a given time; 2 ) manipulating joint angles in a way that the squared sum of all joint velocity is minimized; and 3) setting the relative contribution of each joint as determined by the weighting matrix.

Since we have redundant degrees of freedom, we can introduce a secondary objective that determines/ reconfigures the joint angles of the linkage system without changing the end-effector position by using a 
matrix $\left(\mathbf{I}-\mathbf{J}^{\dagger} \mathbf{J}\right)$ which projects an arbitrary vector $\dot{\mathbf{q}}_{0}$ into the null space of $\mathbf{J}$ [12], where $\mathbf{I}$ is an identity matrix. Hence for the manual subsystem:

$$
\dot{\mathbf{q}}=\mathbf{J}_{m}^{\dagger} \dot{\mathbf{p}}_{m}+\left(\mathbf{I}-\mathbf{J}_{m}^{\dagger} \mathbf{J}_{m}\right) \dot{\mathbf{q}}_{0}
$$

Multiplying $\mathbf{J}_{v}$ for on both sides and solving for $\dot{\mathbf{q}}_{0}$ :

$$
\begin{aligned}
& \mathbf{J}_{v} \dot{\mathbf{q}}=\mathbf{J}_{v} \mathbf{J}_{m}^{\dagger} \dot{\mathbf{p}}_{m}+\mathbf{J}_{v}\left(\mathbf{I}-\mathbf{J}_{m}^{\dagger} \mathbf{J}_{m}\right) \dot{\mathbf{q}}_{0} \\
& \dot{\mathbf{q}}_{0}=\left[\mathbf{J}_{v}\left(\mathbf{I}-\mathbf{J}_{m}^{\dagger} \mathbf{J}_{m}\right)\right]^{\dagger}\left(\mathbf{J}_{v} \dot{\mathbf{q}}-\mathbf{J}_{v} \mathbf{J}_{m}^{\dagger} \dot{\mathbf{p}}_{m}\right)
\end{aligned}
$$

By plugging Eq.7 into Eq.6:

$$
\dot{\mathbf{q}}=\mathbf{J}_{m}^{\dagger} \dot{\mathbf{p}}_{m}+\left(\mathbf{I}-\mathbf{J}_{m}^{\dagger} \mathbf{J}_{m}\right)\left[\mathbf{J}_{v}\left(\mathbf{I}-\mathbf{J}_{m}^{\dagger} \mathbf{J}_{m}\right)\right]^{\dagger}\left(\mathbf{J}_{v} \dot{\mathbf{q}}-\mathbf{J}_{v} \mathbf{J}_{m}^{\dagger} \dot{\mathbf{p}}_{m}\right)
$$

which is simplified to Eq.9 with a gain term $(\alpha)$ scaling the secondary objective function [13].

$$
\dot{\mathbf{q}}=\mathbf{J}_{m}^{\dagger} \dot{\mathbf{p}}_{m}+\alpha\left[\mathbf{J}_{v}\left(\mathbf{I}-\mathbf{J}_{m}^{\dagger} \mathbf{J}_{m}\right)\right]^{\dagger}\left(\dot{\mathbf{p}}_{v}-\mathbf{J}_{v} \mathbf{J}_{m}^{\dagger} \dot{\mathbf{p}}_{m}\right)
$$

In the present study, $\alpha$ was set to unity. Then $\mathbf{q}$ is obtained from the numerical integration of $\dot{\mathbf{q}}$, and the drift of the end-effector position was stabilized by a feedback control algorithm [14].

\section{MOTION MEASUREMENTS}

Subjects Ten healthy subjects, $23 \pm 3$ (mean \pm sd) years old with normal vision participated in the experiments.

Equipment $\mathrm{A}$ circular array containing visual targets was placed on the right frontal hemisphere of the subject. The target area extended from 0 (mid-sagittal plane) to $90^{\circ}$ (rightward) at the distance of 60 and $95 \mathrm{~cm}$ from the hip-point (Figure 2A). The height of the target array was either at eye level or $50 \mathrm{~cm}$ below eye level (Figure 2B). Visual targets are distributed over the array at $15^{\circ}$ intervals. Each target was a seven-segment digital display (visual angle $<1^{\circ}$ ).
A

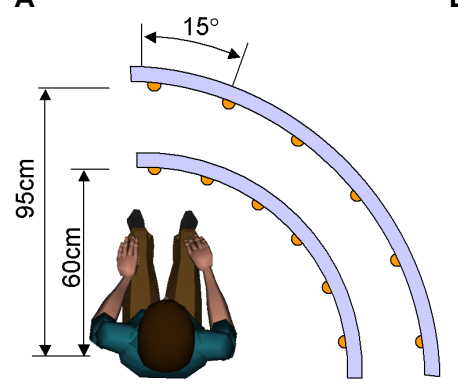

B

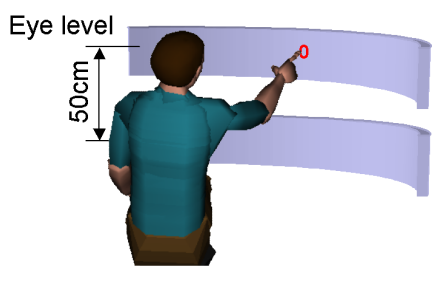

Figure 2. Configuration of targets in a top view $(A)$ and rear view (B).
Procedure In a seated posture, the subjects were asked initially to look at the home target located in the mid-sagittal plane while both hands were resting on the lap (Figure 2A). Then the subject reached with the right hand for a target illuminated at a random location and touched it with the index finger (Figure 2B). Movements of the body links, identified by electromagnetic markers placed on body landmarks, were recorded by a motion capture system (Flock of Birds ${ }^{\mathrm{TM}}$ ).

\section{RESULTS}

Movements were simulated using a model of the manual subsystem alone (Eq. 5), the visual subsystem alone (Eq. 5), or the coordinated manual and visual subsystems (Eq. 9). Examples of superimposed stick figures generated by each model and the corresponding motion measurements are presented in Figure 3. It was observed that all models are capable of predicting the motion trajectories similar to actual motion recordings; however, noticeable differences between predicted and actual motions were found for shoulder and upper arm, and forearm links.

For torso angles, the model of the manual subsystem alone (Figure 4B) made an accurate prediction for $q_{\mathrm{m} 2}$ (lateral bending), while the model of the visual subsystem was better at predicting $q_{m 1}$ (extension) and $q_{m 3}$ (axial rotation). This observation is in agreement with a direct kinematics model (Figure 1) indicating that lateral bending of the torso may not contribute significantly to head rotation in the direction of a target located in the right hemisphere while it may be of primary importance for moving the torso and the hand toward the target. Hence it is suggested that the coordinated model, which benefits from both individual models (manual and visual) provides a better "combined" accuracy for all three torso angles.

In general, the accuracy for eight of the fourteen joint angles of the combined visuo-manual linkage system was significantly improved by the use of a coordinated model (Table 2). Even though $q_{m 1}$ (torso flexion/extension angle) did not show a significant difference as a main effect when contrasted by the noncoordinated or coordinated models, the coordination $x$ target height interaction effect indicated that the coordinated model made significantly greater accurate predictions for the low target positions, where downward flexion movements are required for visual gaze and manual reach. Coordination $\times$ target eccentricity interaction effects indicated that prediction accuracy for $q_{m 2}$ (torso lateral bending) increases with target eccentricity in the coordinated model. These results indicate that coordination improves model prediction accuracy when the body segments are effectively involved in a motion. 
A

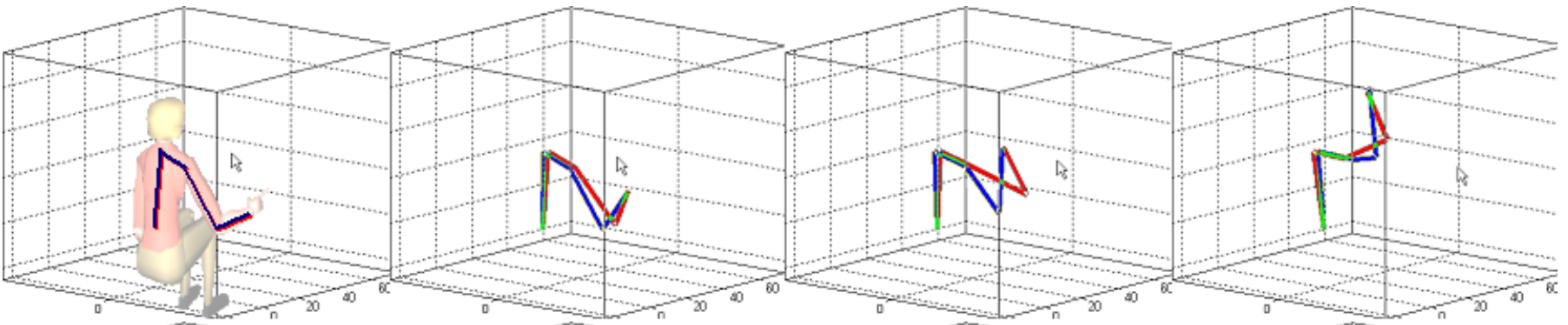

B

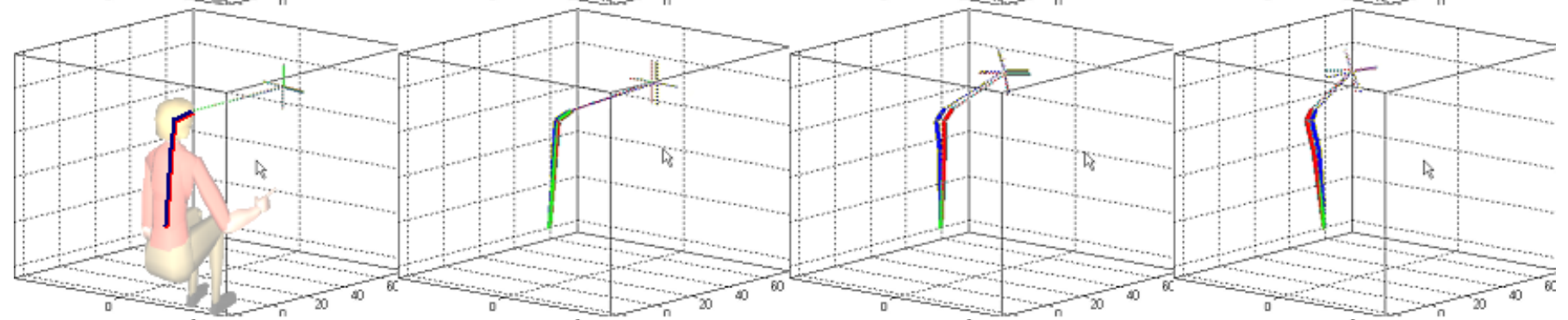

C

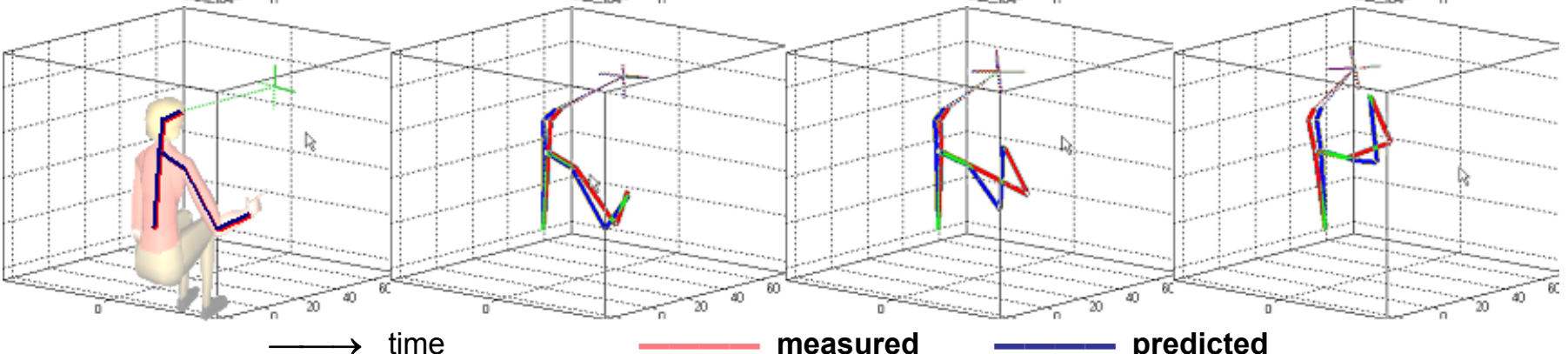

Figure 3. Stick figures generated by actual motion measurements and superimposed model predictions. The target was located in the mid-sagittal plane at the eye level (distance $=60 \mathrm{~cm}$ ). A) manual subsystem; B) visual subsystem; C) manual + visual coordinated. The dotted lines that extend from the cross hairs represent the head orientation vector [15].

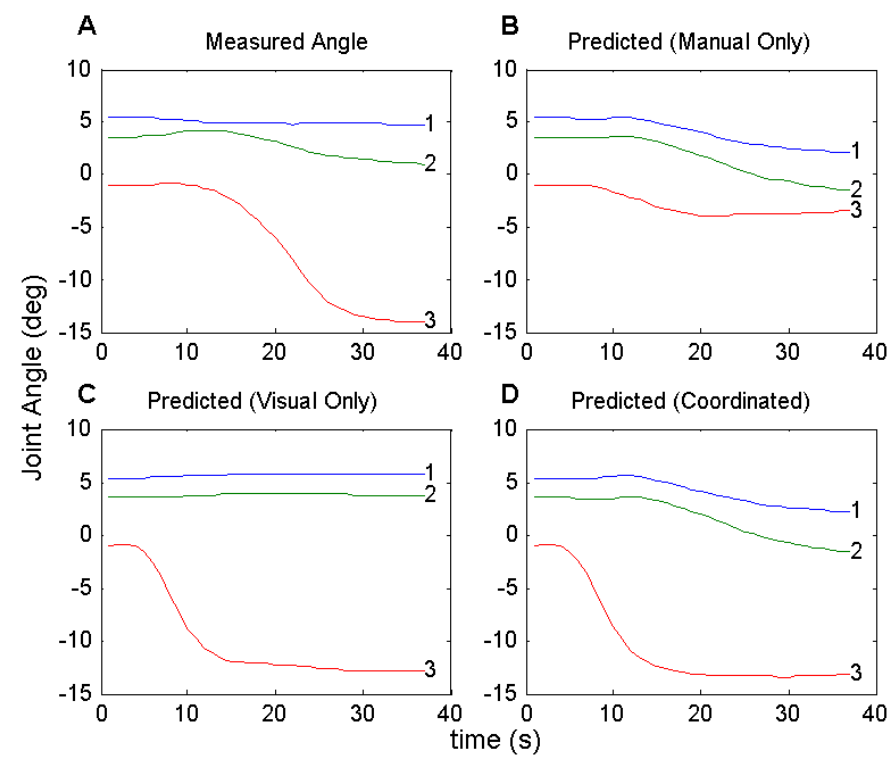

Figure 4. Model simulation result for torso angles; 1 : flexion (up +), 2 : lateral bending (left+), 3: axial rotation (CCW+). Actual angles from motion measurements $(A)$, and predictions by a model of the manual subsystem (B), the visual subsystem (C), and coordinated manual and visual subsystems (D). The target was located at $90^{\circ}$ from the midsagittal plane, $50 \mathrm{~cm}$ below eye level (distance $=60 \mathrm{~cm}$ ).

Table 2. RMS error of the joint angles predicted by models for all target locations. Shaded rows correspond to significant improvements in prediction accuracy by the coordinated model.

\begin{tabular}{lrrl}
\hline Joint & $\begin{array}{c}\text { Non-Coordinated } \\
\left(\text { mean } \pm \mathrm{se}^{\circ}\right)\end{array}$ & $\begin{array}{c}\text { Coordinated } \\
\left(\text { mean } \pm \mathrm{se}^{\circ}\right)\end{array}$ & Significance \\
\hline$q_{m 1}$ & $3.5 \pm 0.3$ & $4.2 \pm 0.4$ & $p<0.05$ \\
$q_{m 2}$ & $2.3 \pm 0.2$ & $3.0 \pm 0.3$ & Non-significant \\
$q_{m 3}$ & $7.7 \pm 0.9$ & $5.1 \pm 0.5$ & $p<0.05$ \\
$q_{m 4}$ & $6.2 \pm 0.7$ & $8.3 \pm 1.0$ & $p<0.05$ \\
$q_{m 5}$ & $8.1 \pm 1.1$ & $7.1 \pm 1.0$ & $p<0.05$ \\
$q_{m 6}$ & $25.4 \pm 2.8$ & $19.5 \pm 2.3$ & $p<0.05$ \\
$q_{m 7}$ & $14.2 \pm 1.6$ & $11.8 \pm 1.1$ & $p<0.05$ \\
$q_{m 8}$ & $39.1 \pm 4.2$ & $35.2 \pm 3.7$ & $p<0.05$ \\
$q_{m 9}$ & $14.7 \pm 0.9$ & $11.8 \pm 1.0$ & $p<0.05$ \\
\hline$q_{v 4}$ & $8.9 \pm 1.0$ & $5.8 \pm 1.0$ & $p<0.05$ \\
$q_{v 5}$ & $4.0 \pm 0.5$ & $5.1 \pm 0.6$ & $p<0.05$ \\
$q_{v 6}$ & $6.5 \pm 0.6$ & $5.3 \pm 0.5$ & $p<0.05$ \\
$q_{v 7}$ & $7.9 \pm 0.3$ & $7.6 \pm 0.4$ & Non-significant \\
$q_{v 8}$ & $4.1 \pm 0.5$ & $4.0 \pm 0.4$ & Non-significant \\
\hline
\end{tabular}


Prediction of end-posture angles was also generally improved by the coordinated model (Table 3). More pronouncedly it was observed that the prediction error for torso axial rotation $\left(q_{m 3}\right)$ was reduced by $85 \%$ when the coordinated model was used. Also the prediction accuracy for $q_{m 1}$ (torso flexion/extension) and $q_{m 2}$ (torso lateral bending) increases for targets at low height and far eccentricity, respectively, which is consistent with the observations about the RMS error statistics above.

Table 3. Error of the joint angles of the end posture predicted by models for all target locations. Shaded rows correspond to significant improvements in prediction accuracy by the coordinated model.

\begin{tabular}{lrrr}
\hline Joint & $\begin{array}{c}\text { Non-Coordinated } \\
\left(\text { mean } \pm \mathrm{se}^{\circ}\right)\end{array}$ & $\begin{array}{c}\text { Coordinated } \\
\left(\text { mean } \pm \mathrm{se}^{\circ}\right)\end{array}$ & Significance \\
\hline$q_{m 1}$ & $0.8 \pm 1.0$ & $2.4 \pm 0.9$ & $p<0.05$ \\
$q_{m 2}$ & $0.7 \pm 0.9$ & $-2.4 \pm 1.0$ & $p<0.05$ \\
$q_{m 3}$ & $11.5 \pm 1.5$ & $1.7 \pm 1.2$ & $p<0.05$ \\
$q_{m 4}$ & $7.9 \pm 2.0$ & $13.0 \pm 2.1$ & $p<0.05$ \\
$q_{m 5}$ & $13.8 \pm 1.8$ & $12.2 \pm 1.8$ & $p<0.05$ \\
$q_{m 6}$ & $-41.0 \pm 4.4$ & $-31.1 \pm 3.7$ & $p<0.05$ \\
$q_{m 7}$ & $24.7 \pm 3.4$ & $14.3 \pm 3.6$ & $p<0.05$ \\
$q_{m 8}$ & $53.3 \pm 10.7$ & $46.3 \pm 10.7$ & $p<0.05$ \\
$q_{m 9}$ & $25.8 \pm 2.0$ & $19.7 \pm 2.4$ & $p<0.05$ \\
\hline$q_{v 4}$ & $-13.6 \pm 1.9$ & $-7.9 \pm 1.8$ & $p<0.05$ \\
$q_{v 5}$ & $2.0 \pm 1.2$ & $6.2 \pm 1.1$ & $p<0.05$ \\
$q_{v 6}$ & $-8.2 \pm 1.4$ & $-3.6 \pm 1.3$ & $p<0.05$ \\
$q_{v 7}$ & $8.2 \pm 1.2$ & $5.7 \pm 1.4$ & $p<0.05$ \\
$q_{v 8}$ & $3.4 \pm 1.0$ & $-2.0 \pm 0.9$ & $p<0.05$ \\
\hline
\end{tabular}

\section{CONCLUSIONS}

In visually guided reach motions, the visual and manual subsystems act to locate the target and move the hand to the target, respectively $[16,17]$. The present model proposes a method of incorporating multiple subsystems with individual end-effectors using an optimization-based inverse kinematics algorithm. In this process of incorporation, coordination is required in order to share common resources and subsystems that are dedicated to manipulate their respective end-effector. The common links may be controlled by either one of the subsystems exclusively, while the other subsystem's control is restrained. Movement accuracy can be viewed as the result of this coordination. Dominance of one subsystem over another may be a function of tasks requirements in a specific context.

It was found that in general the accuracy of the predicted joint angle trajectories was better when the coordination is introduced as a secondary cost function in the differential inverse kinematics. Accordingly the results of this model suggest that 1) the central controller takes into account the constraints of each subsystem to find an optimal set of joint angles, hence coordination can be viewed as the compromise of shared control between identified subsystems involved in a movement; 2) the advantage of the coordination model is more prominent for reach movements to low and eccentric targets. This latter effect shows that the accuracy of the model increases with the effective contribution of a joint to a visually guided reach movement. Hence, accuracy of the coordinated model may be better than it appears when considering only the average RMS errors including all target locations. The statistically significant interaction effects mentioned above support this hypothesis.

\section{ACKNOWLEDGMENTS}

We would like to acknowledge and thank the partners of the Human Motion Simulation project for their support of the present study (DaimlerChrysler Company, EDS PLM Solutions, Ford Motor Company, General Motors Corporation, International Truck and Engine Corporation, Lockheed Martin Corporation / Sandia National Laboratories, University of Michigan Automotive Research Center, US Army - Tank-Automotive and Armaments Command, and US Postal Service).

\section{REFERENCES}

1. Dysart, M. J., and Woldstad, J. C. 1996, Posture prediction for static sagittal-plane lifting, Journal of Biomechanics, 29(10), 1393-1397.

2. Zhang, X., Kuo, A. D., and Chaffin, D. B. 1999, Optimization-based differential kinematic modeling exhibits a velocity-control strategy for dynamic posture determination in seated reaching movements, Journal of Biomechanics, 31, 10351042.

3. Wang, X., 1999, Behavior-based inverse kinematics algorithm to predict arm prehension postures for computer-aided ergonomic evaluation, Journal of Biomechanics, 32(5), 453-460.

4. Abdel-Malek, K., Yu, W., Jaber, J., and Duncan, J. 2001, Realistic posture prediction for maximum dexterity, SAE Technical Papers Series, 2001-012110 (Warrendale, PA: Society of Automotive Engineers).

5. Kim, K., Martin, B. J., and Park, W. 2000, Head orientation in visually guided tasks, SAE Technical Papers Series, 2000-01-2174 (Warrendale, PA: Society of Automotive Engineers).

6. Delleman, N. J., Huysmans, M. A., and Kujit-Evers, L. F. M. 2001, Postural behaviour in static gazing sidewards, SAE Technical Papers Series, 2001-012093 (Warrendale, PA: Society of Automotive Engineers).

7. Tipper, S. P., Howard, L. A., and Paul, M. A. 2001, Reaching affects saccade trajectories. Experimental Brain Research, 136(2), 241-249.

8. Peterka, R. J., and Benolken, M. S. 1995, Role of somatosensory and vestibular cues in attenuating visually induced human postural sway, Experimental Brain Research, 105(1), 101-110.

9. Cohn, J. V., DiZio, P., and Lackner J. R. 2000, Reaching during virtual rotation: context specific compensations for expected coriolis forces, Journal of Neurophysiology. 83(6), 3230-3240.

10. van der Kooij, H., Jacobs, R., Koopman, B., van der Helm, F. 2001, An adaptive model of sensory 
integration in a dynamic environment applied to human stance control, Biological Cybernetics. 84(2), 103-115.

11. Hashimoto, K. 1999, Observer-based Visual Servoing, In Robotics and Automation. Eds: B. Ghosh, N. Xi and T. J. Tarn, Academic Press, London.

12. Greville, T. N. E. 1959, The pseudoinverse of a rectangular or singular matrix and its application to the solution of systems of linear equations. SIAM Review, 1(1), 38-43.

13. Maciejewski, A. A., and Klein, C. A. 1985, Obstacle avoidance for kinematically redundant manipulators in dynamically varying environments, International Journal of Robotics Research, 4(3), 109-117.

14. Chiacchio, P., Chiaverini, S., Sciavicco, L., and Siciliano, B. 1991, Closed-loop inverse kinematics schemes for constrained redundant manipulators with task space augmentation and task priority strategy. International Journal of Robotics Research, 10, 410-425.

15. Jampel, R. S., and Shi, D. X. 1992, The primary position of the eyes, the resetting saccade, and the transverse visual head plane. Investigative Ophthalmology and Visual Science. 33(8), 25012510.

16. Kim, K. H. and Martin, B. J. 2002, Coordinated Movements of the Head in Hand Reaching Tasks. Proceedings of the 46th Human Factors and Ergonomics Society Conference, Baltimore, Maryland.

17. Vercher, J. L., Magenes, G., Prablanc, C., and Gauthier, G. M. 1994, Eye-head-hand coordination in pointing at visual targets: Spatial and temporal analysis. Experimental Brain Research, 99, 507-523.

\section{CONTACT}

\section{K. Han Kim}

Email: kyunghan@umich.edu

Phone: +1-734-647-3241

Address: 1205 Beal Avenue, Ann Arbor, Ml 48109-2117 USA 